\title{
Cytokines of the Th1 and Th2 type in sera of rheumatoid arthritis patients; correlations with anti-Hsp40 immune response and diagnostic markers
}

\author{
Stefan Tukaj', Agnieszka Kotlarz1', Agnieszka Jóźwik², Żaneta Smoleńska³, Ewa Bryl2, \\ Jacek M. Witkowski² and Barbara Lipińska ${ }^{1 凶}$
}

1Department of Biochemistry, University of Gdańsk, Gdańsk, Poland; 2Faculty and Department of Pathophysiology, and 32Department of Family Medicine, Medical University of Gdańsk, Gdańsk, Poland

\begin{abstract}
Rheumatoid arthritis (RA) is a chronic, systemic inflammatory disease which affects approximately $1 \%$ of the population worldwide. Recent research on the role of heat shock proteins (Hsps) in RA development indicates that they may have pro- or anti-inflammatory effect, most probably via modulating cytokine secretion. We investigated type Th1 (INFY, TNFa, IL-2) and type Th2 (IL10, IL-6, IL-4) cytokine levels in sera of RA patients and healthy controls, using flow cytometric bead array assay, and searched for correlations between the cytokine levels and serum antibodies against bacterial (DnaJ) and human (Hdj1, Hdj2 and Hdj3) Hsp40 proteins, as well as clinical and laboratory parameters. The levels of all cytokines studied were significantly increased in RA patients; the highest increase relative to healthy controls (7-fold) was observed for IL- 6 and its levels correlated positively with the antibodies directed to DnaJ and to the C-terminal domain of $\mathrm{Hdj2}$, and with diagnostic parameters (DAS 28, Steinbrocker RTG criteria, ARA/7, ESR, TEN, SW and GH). INFy levels correlated negatively with DAS 28, ESR, TEN and SW. No correlations were found for TNFa, IL-2 or IL-4. Our results support the hypothesis of Hsp40 involvement in RA as well as indicate that IL-6 serum level is a good marker of the RA activity.
\end{abstract}

Keywords: cytokines, heat shock proteins, Hsp40, rheumatoid arthritis

Received: 28 December, 2010; revised: 06 May, 2010; accepted: 23 August, 2010; available on-line: 09 September, 2010

\section{INTRODUCTION}

Rheumatoid arthritis (RA) is a chronic, systemic inflammatory disease with autoimmune etiology (reviewed by Klareskog et al., 2009). RA affects approx. $1 \%$ of the population worldwide, with the female/male ratio of 2.5/1 (Lee \& Weinblatt, 2001). RA is characterized by persistent and progressive inflammation of peripheral joints, leading to destruction of cartilage and bones (Verhoef, 2001).

Detailed pathogenesis of RA is unknown, however there is strong evidence that immune components, especially $\mathrm{T}(\mathrm{CD} 4+)$ and $\mathrm{B}$ cells are involved in the disease progression (Fournier, 2005). Several functional subtypes of CD4+ $\mathrm{T}$ cells, including Th1, Th2 and regulatory $\mathrm{T}$ cells - Treg, have been described (Skapenko et al., 2005; Shahrara et al., 2008; Bryl et al., 2009). Many reports suggest that RA is mediated by activated pro-inflammato- ry Th1 cells (Schulze-Koops \& Kalden, 2001; Verhoef, 2001) which are characterized mainly by secretion of the IL-2, INF $\gamma$ and TNF $\alpha$ cytokines promoting cellular immune response, whereas Th2 cells produce the IL-4, IL5, IL-6, IL-10 and IL-13 cytokines which stimulate humoral antibody-mediated immune response (Raziuddin et al., 1998). It has been suggested that rheumatoid inflammation is mediated by the dominance of activated Th1 cells producing pro-inflammatory cytokines over the Th2 cells (Verhoef, 2001; Schulze-Koops \& Kalden, 2001).

Heat shock proteins (Hsps) are a family of evolutionarily conserved proteins whose expression at times of cellular stress, including infection and chronic inflammation, is markedly elevated (Zlacka et al., 2006; reviewed by van Eden et al., 2007). Based on their molecular weight, Hsps are classified into protein families, the major being the Hsp100, Hsp70, Hsp90, Hsp60, Hsp40, and small Hsps. Their structure conserved from bacteria to man and high immunogenicity make them attractive targets for investigation in the area of autoimmunity, with Hsp60 and Hsp70 being most extensively studied (reviewed in van Eden et al., 2005; 2007; Wieten et al., 2007). In contrast, the research on Hsp40 involvement in autoimmune diseases, including RA, has been less extensive, despite Hsp40 being probably the largest Hsp family in humans, with at least 50 members (reviewed in Kampinga et al., 2009). Escherichia coli DnaJ is one of the immunogenic bacterial Hsp proteins of the Hsp40 family, of which the Hdj1, Hdj2 and Hdj3 proteins are the best characterized human members (Terada \& Mori, 2000). The Hsp40 proteins comprise a strongly conserved N-terminal domain and a poorly conserved Cterminal region (reviewed in Cheetham \& Caplan, 1998).

Recent studies (Prakken et al., 2002; 2004; Kamphuis et al., 2005; Massa et al., 2007) indicate that recognition of peptides derived from heat shock proteins by the immune system can have an anti-inflammatory effect and down-regulate chronic state of inflammation via modulation of cytokine secretion. Recently, it has been demonstrated that in juvenile rheumathoid arthritis (JIA) peptides derived from bacterial and human Hsp40s modulate autoimmune inflammation (Massa et al., 2007).

\footnotetext{
e-mail: lipinska@biotech.ug.gda.pl
}

Abbreviations: ARA, American Rheumatology Association; DAS 28, disease activity score; ELISA, enzyme-linked immunosorbent assay; ESR, erythrocyte sedimentation rate; $\mathrm{GH}$, general health; INF, interferon; IL, interleukin; PMBCs, peripheral blood mononuclear cells; RA, rheumatoid arthritis; SW, swelling; TEN, tenderness; TNF, tumor necrosis factor 
We found previously that sera of RA patients contained significantly elevated levels of anti-DnaJ, antiHdj2 and anti-Hdj3 antibodies compared to sera of healthy control individuals (Krzewski et al., 2003; Tukaj et al., 2010). Those results suggested an involvement of Hsp40s in RA development.

In the present study, we investigated type Th1 (INF $\gamma$, TNF $\alpha$, IL-2) and type Th2 (IL-10, IL-6, IL-4) cytokine levels in sera of a well characterized group of patients with RA in order to correlate the cytokine levels with anti-Hsp40 humoral response as well as with diagnostic and laboratory parameters of the patients.

\section{MATERIALS AND METHODS}

Patients. Rheumatoid arthritis patients were diagnosed according to American Rheumatism Association (ARA) criteria (Arnett et al., 1987). Forty-eight serum samples of RA patients (46 female and 2 male) (age 46 \pm 16.7 ; range 20-80); disease duration $7.9 \pm 8.5$ (range 0.2-32); RTG Steinbrocker's stages: stage I $(\mathrm{n}=10)$, stage II $(n=10)$, stage III $(n=2)$ and stage IV $(n=14) ; 50 \% R F$ positive patients; ESR $(29.63 \pm 24.33$ mm/h); DAS 28 $(3.81 \pm 1.04))$ from the Pathophysiology Department at the Medical University of Gdańsk and fifty age- and sexmatched healthy volunteers were included in this study. Sera of the RA patients and healthy controls were stored at $-20^{\circ} \mathrm{C}$ prior to evaluation of cytokines and anti-Hsp40 antibodies' levels.

Consent. The study was approved by the Local Committee for Biomedical Research Ethics at the Medical University of Gdańsk. All subjects were informed of the details of the experiment prior to the taking of a sample of $20 \mathrm{ml}$ peripheral venous blood.

Chemicals. Mouse anti-human Fc IgG antibodies coupled with horseradish peroxidase (HRP), the HRP substrate (tetramethylbenzidine) and other chemicals were purchased from Sigma (Poznań, Poland).

Antigens. Hsp40 antigens: bacterial DnaJ, human Hdj1, Hdj2, Hdj3, and N-terminal and C-terminal domains of DnaJ (DnaJ $\Delta 107-375$; DnaJ $\Delta 1$-199) and Hdj2

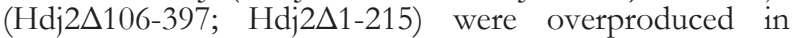

E. coli and purified as described previously (Zylicz et al., 1985; Terada \& Mori, 2000; Krzewski et al., 2003).

Cytokine measurements. Cytokines (INF $\gamma, \mathrm{TNF} \alpha$, IL-6, IL-4, IL-10 and IL-2) were measured in patients' and controls' sera by a flow cytometric bead array using human Th1/Th2 cytokine kit CBA ${ }^{\mathrm{TM}}$ (Becton Dickinson, Poland) and a FACScan (Becton Dickinson).

ELISA assay. Levels of the anti-Hsp40 antibodies ( $\operatorname{IgG}$ ) in the sera of the RA patients and healthy controls were assayed by an indirect ELISA test performed as described before (Krzewski et al., 2003). Briefly, ELISA plates were coated with $50 \mu \mathrm{l}$ of each Hsp40 antigen $(40 \mu \mathrm{g} / \mathrm{ml}$ ) and the sera (diluted 1:500) were added to the wells. Subsequently, mouse anti-human Fc IgG antibodies coupled with horseradish peroxidase were added $(1: 2000)$. After reaction with the HRP substrate tetramethylbenzidine, absorbance was measured at $450 \mathrm{~nm}$ using an ELISA plate reader (Asys Hitech GmbH, Austria). Assays were performed in triplicate.

Statistical analysis. Statistical analyses were performed using the SPSS 12.0 for Windows program (SPSS Inc, Chicago, Il, USA). The distribution normality was analyzed using the Shapiro-Wilk test. Serum cytokine levels were analyzed using Student's $t$-test for unpaired samples. Serum levels of anti-Hsp40 antibodies were analyzed using U Mann Whitney test. Spearman's rank correlation test was used for multi-parametric data with normal (serum cytokine levels), non-normal (serum anti-Hsp40 levels) and diagnostic and laboratory parameters. $P$ values less than 0.05 were considered significant.

\section{RESULTS}

\section{Rheumatoid arthritis patients have elevated serum levels of the Th1 and Th2 cytokines}

Levels of six cytokines representing the Th1 (INF $\gamma$, TNF $\alpha$, IL-2) and Th2 (IL-10, IL-6, IL-4) subpopulations were evaluated in the sera of the RA patients $(n=48)$ and healthy control volunteers $(n=50)$ using cytometric bead array (CBA) technique.

We found that the concentrations of all cytokines tested were significantly higher in the sera of RA patients compared to healthy controls (Fig.
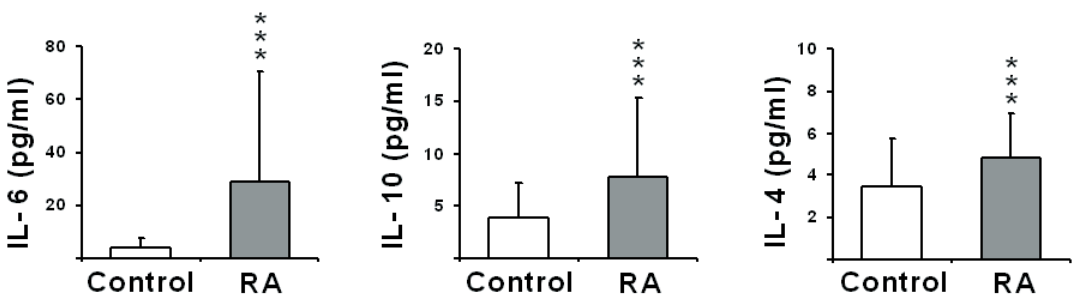
$1)$. The RA/healthy ratio varied from approx. 1.4 for IL-4 to about 7.0 for IL-6. INFy in the sera of RA patients was significantly, approx. 2.5-fold, higher $(P=0.003)$ than in the controls. Interestingly, measurable levels of the cytokines were not found in all RA or healthy sera. Thus, measurable serum levels of INF $\gamma$ were observed in 24/48 RA patients and in $12 / 50$ of the controls, $\mathrm{TNF} \alpha$ in $35 / 48$ sera of RA patients and in $22 / 50$ of the controls, IL-2 in 32/48 RA patients and in $18 / 50$ of the controls, IL-10 in 46/48 RA patients' sera and in 36/50 sera of the controls, and IL-4 were observed in 45/48 RA patients and in $41 / 50$ of the controls. IL- 6
Figure 1. Serum levels of Th1 (INFy, TNFa, IL-2) and Th2 (IL-10, IL-6, IL-4) cytokines of rheumatoid arthritis patients and healthy controls

Bars present mean \pm S.D. (standard deviation) values. Student's $t$-test was used for statistical analysis $* * P \leq 0.01, * * * P \leq 0.001$. 
Table 1. Levels of antibodies against Hsp40 proteins and their domains in sera of RA patients and healthy controls

\begin{tabular}{|c|c|c|c|}
\hline Serum antibody levels & RA patients & Control subjects & U Mann Whitney test results \\
\hline anti-DnaJ & $1.34 \pm 0.29$ & $0.88 \pm 0.22$ & $P<0.001$ \\
\hline anti-N-DnaJ & $0.31 \pm 0.14$ & $0.22 \pm 0.09$ & $P<0.01$ \\
\hline anti-C-DnaJ & $0.13 \pm 0.09$ & $0.11 \pm 0.11$ & $P<0.05$ \\
\hline anti-Hdj1 & $0.54 \pm 0.16$ & $0.59 \pm 0.27$ & n.s. \\
\hline anti-Hdj2 & $2.52 \pm 0.11$ & $1.95 \pm 0.38$ & $P<0.001$ \\
\hline anti-N-Hdj2 & $0.54 \pm 0.09$ & $0.4 \pm 0.12$ & $P<0.001$ \\
\hline anti-C-Hdj2 & $0.6 \pm 0.28$ & $0.51 \pm 0.16$ & n.s. \\
\hline anti-Hdj3 & $1.08 \pm 0.15$ & $0.91 \pm 0.09$ & $P<0.001$ \\
\hline
\end{tabular}

Antibodies (IgG) against E. coli DnaJ, human Hdj1, Hdj2, Hdj3, and N- and C-terminal domains of DnaJ and Hdj2 were measured by ELISA, as described previously (Krzewski et al., 2003). The values are expressed as mean ( \pm S.D.) of A 450/620. To assay significance of the difference between the RA and control antibody levels U Mann Whitney test was used. n.s., not significant.

was detected in all enrolled RA patients' sera (48/48) and in $33 / 50$ of the controls.

The undetectable levels of cytokines were randomly distributed among the patients and no correlations regarding such low levels of cytokines and disease progression could be found.

\section{Correlations between the levels of serum cytokines, anti-Hsp40 response and diagnostic markers}

We assayed antibodies (IgG) directed to E. coli DnaJ (Hsp40), to its human homologs (Hdj1, Hdj2, Hdj3) and to the $\mathrm{N}$ - and C-terminal domains of DnaJ and Hdj2 in sera of RA patients and healthy control individuals using the ELISA method. We found increased levels of the response against DnaJ, Hdj2 and Hdj3 while there was no significant increase in the anti-Hdj1 response in RA patients. The RA patients had higher response to both DnaJ domains and also to the N-terminal domain of Hdj2 compared to healthy controls (Table 1).

We searched for correlations between the levels of cytokines in the sera of the RA patients and the levels of anti-Hsp40 antibodies, as well as the diagnostic parameters. We found positive correlations between the levels of IL-6 and the antibodies directed to DnaJ protein, and to the C-terminal domain of $\mathrm{Hdj} 2$. A negative correlation between the IL-10 levels and the anti-Hdj1 response was observed (Table 2). We found that the levels of

Table 2. Correlations of serum cytokine concentrations with levels of anti-Hsp40 antibodies, and with the clinical and diagnostic parameters of RA patients

\begin{tabular}{|c|c|c|c|c|c|c|}
\hline \multicolumn{7}{|c|}{ Correlations with serum cytokines } \\
\hline Antibodies & IFNY & TNFa & IL-2 & IL-10 & IL-6 & IL-4 \\
\hline Anti-DnaJ & n.s. & n.s. & n.s. & n.s. & $\begin{array}{c}0.289 \\
P=0.046\end{array}$ & n.s. \\
\hline Anti-Hdj1 & n.s. & n.s. & n.s. & $\begin{array}{c}-0.312 \\
P=0.031\end{array}$ & n.s. & n.s. \\
\hline Anti-C-Hdj2 & n.s. & n.s. & n.s. & n.s. & $\begin{array}{c}0.310 \\
P=0.032\end{array}$ & n.s. \\
\hline \multicolumn{7}{|l|}{ Parameters } \\
\hline DAS 28 & $\begin{array}{c}-0.394 \\
P=0.010\end{array}$ & n.s. & n.s. & n.s. & $\begin{array}{c}0.534 \\
P=0.000\end{array}$ & n.s. \\
\hline Steinbrocker & n.s. & n.s. & n.s. & $\begin{array}{c}0.392 \\
P=0.018\end{array}$ & $\begin{array}{c}0.581 \\
P=0.000\end{array}$ & n.s. \\
\hline ARA/7 & n.s. & n.s. & n.s. & n.s. & $\begin{array}{c}0.388 \\
P=0.011\end{array}$ & n.s. \\
\hline ESR & $\begin{array}{c}-0.331 \\
P=0.032\end{array}$ & n.s. & n.s. & n.s. & $\begin{array}{c}0.454 \\
P=0.003\end{array}$ & n.s. \\
\hline TEN & $\begin{array}{c}-0.331 \\
P=0.032\end{array}$ & n.s. & n.s. & n.s. & $\begin{array}{c}0.309 \\
P=0.046\end{array}$ & n.s. \\
\hline SW & $\begin{array}{c}-0.313 \\
P=0.044\end{array}$ & n.s. & n.s. & n.s. & $\begin{array}{c}0.406 \\
P=0.008\end{array}$ & n.s. \\
\hline GH & n.s. & n.s. & n.s. & n.s. & $\begin{array}{c}0.354 \\
P=0.022\end{array}$ & n.s. \\
\hline Age & n.s. & n.s. & n.s. & n.s. & n.s. & n.s. \\
\hline $\mathrm{RF}$ & n.s. & n.s. & n.s. & n.s. & n.s. & n.s. \\
\hline Duration & n.s. & n.s. & n.s. & n.s. & n.s. & n.s. \\
\hline
\end{tabular}

Spearman's rank correlation test was used for statistical analysis. $P$ values less than 0.05 were considered significant. n.s., not significant. 
IL-6, IL-10 and IFN $\gamma$ correlated with diagnostic parameters such as RTG Steinbrocker criteria used to describe progression of radiological changes, DAS 28 index corresponding to activity of RA disease, and four parameters such as ESR, GH, TEN and SW, used to establish the DAS 28 index (Table 2).

\section{DISCUSSION}

In this study we investigated type Th1 (INF $\gamma, \mathrm{TNF} \alpha$, IL-2) and type Th2 (IL-10, IL-6, IL-4) cytokine levels in the sera of a well characterized group of patients with RA (Fig. 1) and searched for correlations between the cytokine levels and serum antibodies against Hsp40 proteins as well as the clinical and laboratory parameters (Table 2).

Of the cytokines tested, we observed the most dramatic differences between the RA and control groups for IL-6. The mean serum concentration of IL-6 was 7-fold higher in RA patients compared to healthy controls (Fig. 1). This is in good agreement with the well established role of this cytokine in the pathogenesis of RA; increased levels of IL-6 have been found in synovial fluid (Houssiau et al., 1988) and in sera (Lacki et al., 1995; 1997). It is postulated that IL-6 stimulates secretion of IL-17 by Th17 cells; this in turn enhances production of cartilage-destructive enzymes and expression of molecules participating in bone destruction (reviewed by Klareskog et al., 2009).

We found increased levels of antibodies to the Hsp40s of bacterial (DnaJ) and human (Hdj2, Hdj3) origin (Table 1 ) in sera of the RA patients. The findings concerning DnaJ are in agreement with the early work of Albani et al. (1995) and with our results obtained for a different group of patients (UK) (Krzewski et al., 2003). The results concerning human Hsp40s are in agreement with our earlier observation (Tukaj et al., 2010). Furthermore, we observed a positive correlation between the levels of IL- 6 and the response to DnaJ and to the C-terminal domain of Hdj2 in RA patients (Table 2). These findings together support the hypothesis of Hsp40s involvement in RA as proposed in early work of Albani et al. (1995). It is tempting to speculate that DnaJ induces the increase in humoral anti-DnaJ response and stimulation of Th2 cells to produce IL-6. This hypothesis is in agreement with our results showing that the presence of DnaJ drastically induced secretion of IL-6 by peripheral blood mononuclear cells (PMBCs) in vitro (Tukaj et al., 2010). Considering the increased levels of both anti-DnaJ antibodies and IL- 6 one should bear in mind that bacterial infection is one of the discussed possible causes of RA and that elevated levels of IL- 6 have been found in peripheral blood in a variety of diseases, including bacterial infections (de Benedetti et al., 1994).

IL-6 correlated positively also with diagnostic parameters such as: disease activity score (DAS 28), Steinbrocker RTG criteria, criteria for diagnosis of RA (ARA/7), and the four parameters for establishing the DAS 28 Index (erythrocyte sedimentation rate — ESR, tenderness - TEN, swelling — SW and general health — GH) (Table 2). These findings confirm that the IL-6 serum level is a very good clinical marker of the RA activity.

Our observation that sera of RA patients contained significantly elevated levels of IL-10 compared to healthy controls (Fig. 1) is in agreement with the work of Lacki et al. (1997). Recently, a significant increase of IL-10 was found only in RA patients hav- ing an increased CRP value (Minami et al., 2006). We did not include CRP assay in our study, however, we found no correlation between IL-10 levels and ESR values. A positive correlation for IL-10 and Steinbrocker RTG criteria was observed (Table 2), which is to some extent surprising, since IL-10 is the main anti-inflammatory cytokine and its administration reduced inflammation in animal models of RA (Groux et al., 2003). However, the high IL-10 levels may represent a counteracting mechanism which is insufficient to balance the production of pro-inflammatory cytokines by the Th1 subpopulation. We found that the IL-10 levels were negatively correlated with the antiHdj1 humoral response (Table 2). It remains to be tested whether Hdj1 has an inhibitory effect on IL-10 secretion by PMBCs.

We found increased levels of INF $\gamma$, which is a proinflammatory cytokine with immunoregulatory and antitumor activity (Billiau, 1996), playing a central role as a mediator of the signs and symptoms of chronic autoimmune inflammation (Schulze-Koops \& Kalden, 2001). INF $\gamma$ was observed to correlate negatively with the disease activity (DAS 28) as well as with three out of the four criteria used to establish the DAS 28 index (ESR, TEN and SW) (Table 2). Previously, Funauchi et al. (1991) found no correlation between INF $\gamma$ serum levels and the clinical findings - the discrepancy with our results could be due to differences between the groups of patients, including variations in their therapies. The negative correlation between INF $\gamma$ and activity of the disease is in agreement with observations that patients treated with INFy showed improvement concerning clinical parameters (Machold et al., 1992), and suggests that INF $\gamma$ plays an important role in maintaining immune homeostasis in patients with RA. Furthermore, our data suggest that its blood level might be a good marker of the RA activity.

TNF $\alpha$ is postulated to play a crucial role in RA development, together with IL-6 and IL-1, and agents blocking its function are effective drugs in RA therapy (reviewed by Klareskog et al., 2009). Our finding that the RA patients had increased TNF $\alpha$ serum levels is in agreement with the above facts, although we were unable to find any correlation between TNF $\alpha$ and the other parameters tested. Previous assessments showed either no significant increase of $\mathrm{TNF} \alpha$ serum levels in RA patients (Fröde et al., 2002) or an increase correlating with systemic inflammation (Altomonte et al., 1992). These discrepancies indicate that TNF $\alpha$ serum level is not a good candidate for the disease marker, probably because of the relatively short half-life of TNF $\alpha$. However, one should remember about the cascade of cytokines, meaning that TNF $\alpha$ induces IL-6 production, and since IL- 6 has a much longer halflife, it is a better marker for practical measurements in patients' sera. The lack of correlation between TNF $\alpha$ levels and the severity of the disease could be also explained by the fact that we assayed TNF $\alpha$ in serum, not in the synovial fluids.

The serum levels of IL- 2 and IL-4 were increased in RA patients but we found no correlations with the antiHsp40 antibodies or clinical and diagnostic parameters (Table 2). Our results concerning IL-4 levels are consistent with a previous report (Rivas et al., 1995) and suggest that IL-4 does not contribute to the deleterious effects of the disease. In the case of IL-2, an earlier report showed a decrease of its serum levels in RA patients (Altamonte et al., 1992). 
Our finding that the levels of the Th2- as well as Th1-type cytokines were increased in RA patients compared to the healthy controls shows that the serum levels of cytokines do not simply reflect the theory of the dominance of the Th1 over the Th2 response in RA, the theory which is based on the research on synovium (Verhoef, 2001; Schulze-Koops \& Kalden, 2001).

\section{CONCLUSION}

The existence of a positive correlation between the serum levels of IL-6 and antibodies against bacterial Hsp40 (E. coli DnaJ and C-terminal domain of human Hdj2) in RA patients supports the hypothesis of Hsp40 involvement in RA. The increased levels of both Th1- and Th2type cytokines show that the serum levels of cytokines do not simply reflect the theory of the dominance of the Th1 over the Th2 response in RA.

\section{Acknowledgements}

This work was supported by grants from the Ministry of Science and Higher Education (No. 1610/B/ P01/2008/35) and from the University of Gdańsk (BW/1460-5-0380-8).

\section{REFERENCES}

Albani S, Keystone EC, Nelson JL, Ollier WE, La Cava A, Montemayor AC, Weber DA, Montecucco C, Martini A, Carson DA (1995) Positive selection in autoimmunity: abnormal immune responses to a bacterial dnaJ antigenic determinant in patients with early rheumatoid arthritis. Nat Med 1: 448-452.

Albani S, Carson DA (1996) A multistep molecular mimicry hypothesis for the pathogenesis of rheumatoid arthritis. Immunol Today 17: $466-470$.

Altomonte L, Zoli A, Mirone L, Scolieri P, Magaró M (1992) Serum levels of interleukin-1b, tumour necrosis factor- $\alpha$ and interleukin-2 in rheumatoid arthritis. Correlation with disease activity. Clin Rheumatol 11: 202-205.

Arnett FC, Edworthy SM, Bloch DA, McShane DJ, Fries JF, Cooper NS, Healey LA, Kaplan SR, Liang MH, Luthra HS et al. (1988) The American Rheumatism Association 1987 revised criteria for the classification of rheumatoid arthritis. Arthritis Rheum 31: 315-324.

Billiau A (1996) Interferon- $\gamma$ : biology and role in pathogenesis. Adv Immunol 62: 61-130.

Bryl E, Daca A, Jóźwik A, Witkowski JM (2009) Human CD4(low) CD25(high) regulatory $\mathrm{T}$ cells indiscriminately kill autologous activated T cells. Immunology doi: 10.1111/j.1365-2567.20081.x

Cheetham ME, Caplan AJ (1998) Structure, function and evolution of DnaJ: conservation and adaptation of chaperone function. Cell Stress Chaperones 3: 28-36.

De Benedetti F, Massa M, Pignatti P, Albani S, Novick D, Martini AJ (1994) Serum soluble interleukin 6 (IL-6) receptor and IL-6/soluble IL-6 receptor complex in systemic juvenile rheumatoid arthritis. $J$ Clin Invest 93: 2114-2119.

van Eden W, van der Zee R, Prakken B (2005) Heat-shock proteins induce T-cell regulation of chronic inflammation. Nat Rev Immunol 5: 318-330.

van Eden W, Wick G, Albani S, Cohen I (2007) Stress, heat shock proteins, and autoimmunity: how immune responses to heat shock proteins are to be used for the control of chronic inflammatory diseases. Ann N Y Acad Sci 1113: 217-237.

Funauchi M, Sugishima H, Minoda M, Horiuchi A (1991) Tohoku Serum level of interferon-gamma in autoimmune diseases. J Exp Med 164: $259-267$.

Fournier C (2005) Where do T cells stand in rheumatoid arthritis? Joint Bone Spine 72: 527-532.

Fröde TS, Tenconi P, Debiasi MR, Medeiros YS (2002) Tumour necrosis factor-alpha, interleukin-2 soluble receptor and different inflammatory parameters in patients with rheumatoid arthritis. Mediators Inflamm 11: 345-349.

Groux H, Cottrez F, Houssiau FA, Devogelaer JP, Van Damme J., de Deuxchaisnes CN, Van Snick J (2003) The complex role of interleukin-10 in autoimmunity. J Autoimmun 20: 281-285.
Houssiau FA, Devogelaer JP, Van Damme J, de Deuxchaisnes CN, Van Snick J (1988) Interleukin-6 in synovial fluid and serum of patients with rheumatoid arthritis and other inflammatory arthritides. Arthritis Rheum 31: 784-788.

Kampinga HH, Hageman J, Vos MJ, Kubota H, Tanguay RM, Bruford EA, Cheetham ME, Chen B, Hightower LE (2009) Guidelines for the nomenclature of the human heat shock proteins. Cell Stress Chaperones 14: 105-111.

Kamphuis S, Kuis W, de Jager W, Teklenburg G, Massa M, Gordon G, Boerhof M, Rijkers GT, Uiterwaal CS, Otten HG, Sette A, Albani S, Prakken BJ (2005) Tolerogenic immune responses to novel T-cell epitopes from heat-shock protein 60 in juvenile idiopathic arthritis. Lancet 366: 50-56.

Klareskog L, Catrina AI, Paget S (2009) Rheumatoid arthritis. Lancet 373: 659-672.

Krzewski K, Kunikowska D, Wysocki J, Kotlarz A, Thompkins P, Ashraf W, Lindsey N, Picksley S, Glosnicka R, Lipinska B (2003) Characterization of the anti-DnaJ monoclonal antibodies and their use to compare immunological properties of DnaJ and its human homologue HDJ-1. Cell Stress Chaperones 8: 8-17.

Lacki JK, Klama K, Mackiewicz SH, Mackiewicz U, Müller W (1995) Circulating interleukin 10 and interleukin-6 serum levels in rheumatoid arthritis patients treated with methotrexate or gold salts: preliminary report. Inflamm Res 44: 24-26.

Lacki JK, Samborski W, Mackiewicz SH (1997) Interleukin-10 and interleukin-6 in lupus erythematosus and rheumatoid arthritis, correlations with acute phase proteins. Clin Rheumatol 16: 275-278.

Lee DM, Weinblatt ME (2001) Rheumatoid arthritis. Lancet 358: 903 911.

Machold KP, Neumann K, Smolen JS (1992) Recombinant human interferon gamma in the treatment of rheumatoid arthritis: double blind placebo controlled study. Ann Rheum Dis 51: 1039-1043.

Massa M, Passalia M, Manzoni SM, Campanelli R, Ciardelli L, Yung GP, Kamphuis S, Pistorio A, Meli V, Sette A, Prakken B, Martini A, Albani S (2007) Differential recognition of heat-shock protein dnaJ-derived epitopes by effector and Treg cells leads to modulation of inflammation in juvenile idiopathic arthritis. Arthritis Rheum 56: $1648-1657$.

Minami R, Sakai K, Mivamura T, Yamamoto M, Suematsu E (2006) The role of CD4+CD25+ regulatory $\mathrm{T}$ cells in patients with rheumatoid Arthritis. Nibon Rinsho Meneki Gakkeai Kaishi 29: 37-42.

Prakken B, Kuis W, van Eden W, Albani S (2002a) Heat shock proteins in juvenile idiopathic arthritis: keys for understanding remitting arthritis and candidate antigens for immune therapy. Curr Rheumatol Rep 4: 466-473.

Prakken BJ, Roord S, van Kooten PJ, Wagenaar JP, van Eden W, Albani S, Wauben MH (2002b) Inhibition of adjuvant-induced arthritis by interleukin-10-driven regulatory cells induced via nasal administration of a peptide analog of an arthritis-related heat-shock protein 60 T cell epitope. Arthritis Rheum 46: 1937-1946.

Prakken BJ, Samodal R, Le TD, Giannoni F, Yung GP, Scavulli J, Amox D, Roord S, de Kleer I, Bonnin D, Lanza P, Berry C, Massa M, Billetta R, Albani S (2004) Epitope-specific immunotherapy induces immune deviation of proinflammatory $T$ cells in rheumatoid arthritis. Proc Natl Acad Sci USA 101: 4228-4233.

Raziuddin S, Bahabri S, Al-Dalaan A, Siraj AK, Al-Sedairy S (1998) A mixed Th1/Th2 cell cytokine response predominates in systemic onset juvenile rheumatoid arthritis: immunoregulatory IL-10 function. Clin Immunol Immunopathol 86: 192-198.

Rivas D, Mozo L, Zamorano J, Gayo A, Torre-Alonso JC, Rodríguez A, Gutiérrez CJ (1995) Upregulated expression of IL-4 receptors and increased levels of IL-4 in rheumatoid arthritis patients. Autoimmun 8: $587-600$.

Schulze-Koops H, Kalden JR (2001) The balance of Th1/Th2 cytokines in rheumatoid arthritis. Best Pract Res Clin Rheumatol 15: 6776791.

Shahrara S, Huang Q, Mandelin AM, Pope RM (2008) TH-17 cells in rheumatoid arthritis. Arthritis Res Ther 10: R93.

Skapenko A, Leipe J, Lipsky PE, Schulze-Koops H (2005) The role of the $\mathrm{T}$ cell in autoimmune inflammation. Arthritis Res Ther 2: 4-14.

Terada K, Mori M (2000) Human DnaJ homologs dj2 and dj3, and bag-1 are positive cochaperones of hsc70. J Biol Chem 275: $24728-$ 24734.

Tukaj S, Kotlarz A, Jozwik A, Smolenska Z, Bryl E, Witkowski JM, Lipinska B (2010) Hsp40 proteins modulate humoral and cellular immune response in rheumatoid arthritis patients. Cell Stress Chaperones. 15: 555-566. Epub 2010 Feb 2.

Uchiyama Y, Yoshida H, Koike N, Hayakawa N, Sugita A, Nishimura T, Mihara M (2008) Anti-IL-6 receptor antibody increases blood IL-6 level via the blockade of IL-6 clearance, but not via the induction of IL-6 production. Immunopharmacol 8: 1595-601.

Verhoef C (2001) T1/T2 cell balance in rheumatoid arthritis. Clin Appl Immunol Rev 1: 153-161.

Wieten L, Broere F, van der Zee R, Koerkamp EK, Wagenaar J, van Eden W (2007) Cell stress induced HSP are targets of regulatory 
T cells: a role for HSP inducing compounds as anti-inflammatory immuno-modulators? FEBS Lett 581: 3716-3722.

Zlacka D, Vavrincova P, Hien Nguyen TT, Hromadnikova I (2006)

Frequency of anti-hsp60, -65 and -70 antibodies in sera of patients

with juvenile idiopathic arthritis. J Autoimmun 27: 81-88.
Zylicz M, Yamamoto T, McKittrick N, Sell S, Georgopoulos C (1985) Purification and properties of the dnaJ replication protein of Escherichia coli. J Biol Chem 260: 7591-758. 